\title{
Non-Newtonian blood flow in human right coronary arteries: Transient simulations*
}

\author{
Barbara M. Johnston and Peter R. Johnston ${ }^{\dagger}$ \\ School of Science \\ Griffith University \\ Kessels Rd \\ Nathan \\ Queensland \\ Australia 4111 \\ Phone: 61-7-3875-7748 \\ Fax: 61-7-3875-7656 \\ E-mail: P.Johnston@.griffith.edu.au \\ Stuart Corney and David Kilpatrick \\ Department of Medicine \\ University of Tasmania \\ GPO Box 252-34 \\ Hobart \\ Tasmania \\ Australia 7001
}

November 25, 2004

\footnotetext{
*This work was funded, in part, by the National Health and Medical Research Council of Australia and the Griffith University Research and Development Scheme.

${ }^{\dagger}$ Corresponding author
} 


\begin{abstract}
This study looks at pulsatile blood flow through four different right coronary arteries, which have been reconstructed from bi-plane angiograms. A non-Newtonian blood model (the Generalised Power Law), as well as the usual Newtonian model of blood viscosity, is used to study the wall shear stress in each of these arteries over the entire cardiac cycle. The difference between Newtonian and non-Newtonian blood models is also studied over the whole cardiac cycle using the recently generalised global non-Newtonian importance factor. In addition, the flow is studied by considering paths of massless particles introduced into the flow field.

The study shows that, when studying the wall shear stress distribution for transient blood flow in arteries, the use of a Newtonian blood model is a reasonably good approximation. However, to study the flow within the artery in greater detail, a non-Newtonian model is more appropriate.
\end{abstract}

Keywords: non-Newtonian, blood flow, transient, right coronary artery, computational fluid dynamics 


\section{Introduction}

This paper presents a transient simulation study of blood flow in a variety of human right coronary arteries for different models of blood viscosity. It is a companion paper to a recent paper (Johnston et al., 2004) describing the results of steady flow simulations in the same arteries using an even larger number of blood viscosity models.

The idea behind this paper is to compare the effects of different blood viscosity models on the wall shear stress distributions in the right coronary artery during the cardiac cycle. This comparison will allow us to assess whether or not it is necessary to include non-Newtonian blood models in modelling blood flow in coronary arteries.

The flow of blood within arteries has long been associated with the formation of lesions and, eventually, arterial narrowing. Experimental and numerical investigation of these relationships has been the subject of recent reviews (Caro, 2001; Liepsch, 2002). Much of the early numerical modelling was focused on the carotid artery bifurcation (Perktold and Resch, 1990; Perktold et al., 1991b; Perktold et al., 1991a), but, recently, this focus has shifted to the right coronary artery, both in terms of simulation (Krams et al., 1997; Kirpalani et al., 1999; van Langenhove et al., 2000; Myers et al., 2001; Wentzel et al., 2003; Zeng et al., 2003) and experimentally (Krams et al., 1997; Ojha et al., 2001; Feldman et al., 2002; Zhu et al., 2003).

It is well known that blood is a non-Newtonian fluid (Pedley, 1980; Berger and Jou, 2000) and several models have been proposed to predict the stress-strain relationship for blood (Walburn and Schneck, 1976; Cho and Kensey, 1991; Fung, 1993; Ballyk et al., 1994). However, none of these models is generally accepted as a reflection of the true behaviour of the rheology of blood. This is, perhaps, the reason why blood is most often assumed to be a Newtonian fluid in simulations of blood flow. It is also generally accepted that blood behaves as a Newtonian fluid at shear rates above $100 \mathrm{~s}^{-1}$ (Pedley, 1980; Berger and Jou, 2000), which may account 
for the Newtonian approximation in flow simulations at larger Reynolds numbers. However, in transient studies, especially when the flow stops, there are periods of time where the shear rate is below $100 \mathrm{~s}^{-1}$ (Pedley, 1980) and non-Newtonian effects could become important.

In the companion paper (Johnston et al., 2004), it was shown that non-Newtonian effects are important in steady flow simulations, especially for low flow velocities. Since, during the cardiac cycle there are periods of slow flow, it would be reasonable to expect that non-Newtonian effects are important during these periods. The question is, however, to what extent are these effects important over an entire cardiac cycle?

There does not appear to be a consensus in the literature on the importance of nonNewtonian effects on unsteady flows in large arteries. Some studies found non-Newtonian rheology important (Rodkiewicz et al., 1990; Tu and Deville, 1996; Gijsen et al., 1999), while others found that it is relatively unimportant (Perktold et al., 1989; Ballyk et al., 1994), in determining flow patterns in large arteries. The paper of Ballyk et al. (Ballyk et al., 1994) contains a good discussion of possible reasons for this discrepancy, particularly in light of their definition of the non-Newtonian importance factor. A more recent comparison has been conducted for flow through a $90^{\circ}$ curved tube (Gijsen et al., 1999) which highlights several differences between Newtonian and non-Newtonian flow patterns. The paper also describes the calculation of an effective Newtonian viscosity which captures the non-Newtonian effects for this flow situation.

Comparisons between the various arteries and blood viscosity models are presented in terms of wall shear stress distributions as it is believed that wall shear stress is a significant factor in the onset of coronary artery disease (Fry, 1968; Caro et al., 1971; Giddens et al., 1990; Ku et al., 1985). Further comparisons are presented in terms of the recently generalised (Johnston et al., 2004) local and global non-Newtonian importance factors (Ballyk et al., 1994).

It is worth noting that the various non-Newtonian blood models are obtained by parameter 
fitting to experimental viscosity data obtained at certain shear rates under steady state conditions (Walburn and Schneck, 1976; Cho and Kensey, 1991; Fung, 1993; Ballyk et al., 1994). Here, it is assumed that these models can be used under pulsatile flow conditions. This assumption is a possible limitation to this work, since the reformation of red blood cell rouleaux is different under steady and pulsatile conditions. In fact, rouleaux formation in pulsatile larger artery flow is not very likely to occur (Gijsen et al., 1999). The work here, however, involves a comparison of wall shear stress distributions, which are obtained using both the Newtonian and non-Newtonian models of blood viscosity. So, while major differences between the results of these two models would need to be examined in the light of the above comments, similar results for the models would indicate that this assumption is most likely a reasonable one.

\section{Background}

Much of the background to this work has been presented in the companion paper on steady flows (Johnston et al., 2004). However, the main ideas will be briefly recapped here.

Images of the right coronary artery were collected from patients during routine angiography. Four sets of angiograms (labelled A to D) were chosen because there was no sign of atheroma in the arteries. These arteries have been described in detail previously (Johnston et al., 2004),

with arteries A, B and D representing normal arteries with diameters from 3 to $5 \mathrm{~mm}$ and artery $\mathrm{C}$ representing a so-called 'ectatic' artery with diameters from 5 to $7 \mathrm{~mm}$. The arteries were recorded as biplane angiograms (Figure 1 shows the angiograms for artery B as an example), and, using a previously published technique (Corney et al., 2001; Corney et al., 2004) based on centreline extraction and edge detection, the arteries were reconstructed in three dimensions resulting in a mesh suitable for computational fluid dynamics simulation. Figure 2 shows the variation in arterial radius (calculated as a geometric mean of the radii for each view) 
as a function of distance along the artery. It is clear from this figure that the arteries vary considerably in their geometry.

As detailed in (Johnston et al., 2004), five non-Newtonian models for blood viscosity were considered in the flow simulations as well as a Newtonian model. Given the discussion and results in (Johnston et al., 2004), it was decided that here comparisons would only be made between the Generalised Power Law and Newtonian models. For the Generalised Power Law (Ballyk et al., 1994) model, the blood viscosity $\mu$, in Poise, as a function of strain $(\dot{\gamma})$ is given by:

$$
\mu=\lambda(\dot{\gamma})|\dot{\gamma}|^{n(\dot{\gamma})-1}
$$

where

$$
\begin{aligned}
& \lambda(\dot{\gamma})=\mu_{\infty}+\Delta \mu \exp \left[-\left(1+\frac{|\dot{\gamma}|}{a}\right) \exp \left(\frac{-b}{|\dot{\gamma}|}\right)\right] \\
& n(\dot{\gamma})=n_{\infty}-\Delta n \exp \left[-\left(1+\frac{|\dot{\gamma}|}{c}\right) \exp \left(\frac{-d}{|\dot{\gamma}|}\right)\right]
\end{aligned}
$$

with $\mu_{\infty}=0.0345, n_{\infty}=1.0, \Delta \mu=0.25, \Delta n=0.45, a=50, b=3, c=50$ and $d=4$. This Generalised Power Law model fits experimental stress-strain measurements over the range of strain rates, $\dot{\gamma}, 0.1<\dot{\gamma}<1000 s^{-1}$ (Ballyk et al., 1994). The model also encapsulates the behaviours of many of the other blood models. Specifically, it behaves like the Power Law model at low strain rates, is Newtonian at high strain rates and has Casson and Carreau models as special cases.

Finally, comparisons are presented in terms of wall shear stress and the local, $I_{L}$, and global, $\bar{I}_{G}$, non-Newtonian importance factors. These were defined in (Johnston et al., 2004) as

$$
I_{L}=\frac{\mu}{\mu_{\infty}}
$$

and

$$
\bar{I}_{G}=\frac{1}{N} \frac{\left[\sum_{N}\left(\mu-\mu_{\infty}\right)^{2}\right]^{\frac{1}{2}}}{\mu_{\infty}} \times 100
$$


where $\mu$ is the non-Newtonian viscosity (in Poise) and $\mu_{\infty}=0.0345 P$ is the Newtonian viscosity of blood. Note that $\bar{I}_{G}$ is evaluated only at each of the $N$ nodes on the surface mesh of the artery. As mentioned above, these quantities are generalisations of similar ideas described in Ballyk et al. (Ballyk et al., 1994).

\section{Modelling}

It will be assumed that the flow of blood in the right coronary artery is governed by the Navier-Stokes equations

$$
\rho\left(\frac{\partial \mathbf{v}}{\partial t}+\mathbf{v} \cdot \nabla \mathbf{v}\right)=-\nabla \cdot \tau-\nabla P
$$

and the continuity equation for an incompressible fluid

$$
\nabla \cdot \mathbf{v}=0
$$

In these equations, $\mathbf{v}$ is the three dimensional velocity vector, $t$ time, $P$ pressure, $\rho$ density and $\tau$ the stress tensor. Writing the Navier-Stokes equations in this form allows the flexibility to use an arbitrary non-Newtonian blood model.

As described previously, (Corney et al., 2001; Johnston et al., 2004; Corney et al., 2004), coronary artery meshes were reconstructed from biplane angiograms, from which the centreline and radius of the artery along the centreline were extracted. The mesh was completed by creating a mesh of the entrance plane and extruding this mesh along the centreline, taking into account variations in radius. As an example, the mesh for artery B is shown in Figure 3. With the artery reconstructed in this fashion, the governing equations can be solved using the finite volume method. The equations were solved using the commercially available software package CFD-ACE (CFDRC, Huntsville, Alabama, USA) for performing computational fluid dynamics simulations. 
The boundary conditions required to solve the governing equations are as follows. On the solid walls of the artery (which are assumed to be rigid), the no-slip condition is imposed on the velocities. At the outlet of the artery, the velocities are free, but the normal and tangential shear stresses are constrained to be zero and the gauge pressure is also set to zero. At the inlet of the artery a uniform inlet velocity profile with the time varying forcing function (or velocity multiplier) given in Figure 4 is assumed (Matsuo et al., 1988, Figure 4A). This physiological profile was recorded in the right coronary artery of a normal 56-year-old female and contains some periods of reverse flow as well as periods of rapid acceleration and deceleration. The flow pattern is characterised by a small forward flow during systole ( $\mathrm{S}$ wave) with a large forward flow during diastole (D wave) (Matsuo et al., 1988). In this particular case, the peak of the $\mathrm{S}$ wave is just below half the height of the $\mathrm{D}$ wave. The forcing function was scaled to yield a maximum inflow velocity of $0.214 \mathrm{~ms}^{-1}$ with a heart rate of approximately 60 beats per minute.

The simulations presented here are representative of a cyclic phenomenon in time, hence it is expected that the solution at the beginning of the cycle should be identical to the solution at the end of the cycle. To achieve this, the model was initially configured with flow data from a steady state simulation with an inlet velocity of $0.1 \mathrm{~ms}^{-1}$. It was then run over a number of cycles until the solution at the start of the cycle was identical to that at the end of the cycle. Typically, transient runs over five cycles were sufficient to satisfy this condition.

One approximation used in this modelling is that the same mesh is used throughout the cardiac cycle. That is, flow is simulated with a realistic velocity forcing function but on a fixed mesh. Therefore, the fact that the right coronary artery changes shape as the heart beats has been ignored, which is a reasonable first approximation (Zeng et al., 2003). Also, despite the fact that flow rate ratios between main and daughter branches have been shown to have a significant effect on wall shear stress distributions (Asakura and Karino, 1990; Perktold et al., 1991b), this study does not include the side-branches of the right coronary artery. A partial 
justification for this is that the right coronary artery generally has fewer side branches than the left coronary artery (Asakura and Karino, 1990) and also that other studies of flow in the right coronary artery have ignored side branches (Krams et al., 1997; Berthier et al., 2002; Zeng et al., 2003).

\section{Results}

Transient simulations were performed for each of the four arteries described above. Each simulation was from $t=0$ to $t=1.00$ second, yielding a heart rate of approximately 60 beats per minute. For all four arteries, the simulations were performed using the Generalised Power Law blood viscosity model with additional simulations performed using a Newtonian model for arteries B and C. These two arteries were chosen because B is representative of the normal arteries and $\mathrm{C}$ is an ectatic artery. These two simulations are used to study the difference between the two blood viscosity models.

Figure 5 shows a representative data set of the wall shear stress distributions in artery B using the Generalised Power Law blood viscosity model. Distributions are shown at the time values of $0.01,0.13,0.18,0.35,0.72$ and 0.85 seconds through the cardiac cycle. These values were chosen as they are representative of key points during the cardiac cycle:

- $t=0.01$ is near the beginning of the cycle, just prior to the deceleration of the blood flow;

- $t=0.13$ is the point of maximum reverse velocity;

- $t=0.18$ is where the flow has stopped prior to the acceleration;

- $t=0.35$ is at the peak of the $\mathrm{S}$ wave;

- $t=0.72$ is approximately half way through the rapid acceleration phase. 
- $t=0.85$ corresponds to the point of maximum forward velocity, the peak of the D wave;

At this point it is worth mentioning that there is very little visual difference in wall shear stress distribution between the two blood viscosity models throughout the cardiac cycle.

\subsection{Wall Shear Stress Distributions}

Figure 5 shows a set of very complex patterns of wall shear stress in artery B. In each panel of this figure, the small inset in the upper right of the panel shows an enlarged version of the wall shear stress distribution on the reverse side of the artery, in the square shown on the main figure. At the beginning $(t=0.01)$ of the cycle there are quite marked regions of low wall shear stress at the proximal end of the artery and further patches of low wall shear stress at various intervals along the artery. Two intense regions of low wall shear stress appear on the back of the artery. For this particular wave form (Figure 4), the magnitude of the peak (at $t=0.13)$ reverse flow is not particularly large and so the wall shear stress is uniformly low over the entire artery. At the point where the flow has stopped $(t=0.18)$, the wall shear stress is again uniformly low, but lower than that at $t=0.13$. At the peak of the $\mathrm{S}$ wave $(t=0.35)$, the wall shear stress distribution is almost identical to that at $t=0.01$, which is perhaps not surprising as the velocity is about the same. However, the regions of low shear do not appear as low as when $t=0.01$, in other words, spatial gradients of wall shear stress do not seem as high. During rapid acceleration $(t=0.72)$ patches of moderate wall shear stress appear and regions of low wall shear stress remain. The regions of moderate wall shear stress occur just below the first main bend at the proximal end of the artery and on the inner bends of the artery further downstream. At the peak velocity $(t=0.85)$, the regions of moderate wall shear stress have intensified to high wall shear stress and regions of low wall shear stress towards the proximal end of the artery have also intensified. As the flow decelerates, the regions of high wall shear 
stress begin to dissipate and more regions of low wall shear stress begin to appear. Finally, as time continues, the pattern at $t=0.01$ re-emerges. A full movie showing this simulation is available from the Journal's web site.

In these complex patterns of wall shear stress throughout the cardiac cycle, there are several regions in the proximal part of the artery which show low wall shear stress at all times. These regions include the point on the outside wall of the artery, indicated by the boxes in each panel of Figure 5, as well as two points which are either side of the point on the inside wall opposite to that point, as indicated in the insets in the panels.

In these regions the wall shear stress is always less than approximately $1 \mathrm{~Pa}$ and, because of this low value, it might be expected that a non-Newtonian viscosity model would be required.

The above description of the wall shear stress distribution throughout the cardiac cycle is typical of the other three arteries considered (again movies and further figures are available on the Journal's web site). In general, the regions of high wall shear stress at high velocities are predominantly near the distal ends of the artery. Yet for all arteries, there are regions of low wall shear stress near the proximal end at all points of the cardiac cycle. These regions of low wall shear stress also exist towards the distal end of artery C.

To demonstrate the above comments at one particular point of the cardiac cycle, Figure 6 shows wall shear stress distributions for all four arteries at $t=0.85 \mathrm{sec}$, the point where the flow is at a maximum. Note that the range of shear stress in this figure varies from 0 to $10 \mathrm{~Pa}$, as opposed to 0 to $4 \mathrm{~Pa}$ in Figure 5. Artery A shows two small points of high wall shear stress in the first third of the artery (predominantly on the opposite side, one of which is shown in the inset), and several more regions at the distal end of the artery. There is a large region of low wall shear stress midway along the artery which corresponds to the widening of the artery (Figure 2). Artery B shows a much narrower range of wall shear stress, all in the low to mid ranges of the given scale. From Figure 2, it can be seen that the radius of artery B is almost 
constant. The ectatic artery, artery $\mathrm{C}$, also shows predominantly low to medium wall shear stress, except for one point where there is high wall shear stress, approximately where there is a narrowing in the artery radius profile, Figure 2. Finally, artery D shows gradually increasing wall shear stress from proximal to distal ends, due to the more-or-less constant taper of the artery (Figure 2). The highest wall shear stress is towards the outside of the artery, distal to the final bend. One factor common to arteries A, C and D is the presence of high wall shear stress gradients near the regions of maximal wall shear stress, which can be observed in the inset figures for each artery. The above description is common to most of the cardiac cycle where the flow is moving from the proximal to distal ends.

\subsection{Non-Newtonian Importance Factor}

Figure 7 shows the local non-Newtonian importance factor $\left(I_{L}\right)$ for artery B at various points during the cardiac cycle. It can be seen that for most of the cycle, the non-Newtonian behaviour of blood is not important. The only time at which it is significant is during the period when the flow is decelerating and close to zero flow, during the $0.05-0.25$ second period of the cycle. It is interesting to observe from Figure 7 that there is a difference in $I_{L}$ distributions when $t=0.01$ and $t=0.35$, despite the fact that at these times the wall shear stress distribution is similar (Figure 5). At $t=0.01$ the $I_{L}$ distribution shows three peaks on the reverse side of the artery, whereas at $t=0.35$, these peaks do not exist. The frames of figure 7 are fairly typical of the results for the other arteries considered, except that in arteries $\mathrm{A}$ and $\mathrm{D}$ the distribution of $I_{L}$ at $t=0.18$ is almost entirely red and for artery $\mathrm{C}$ at $t=0.18$, there is a greater variation in $I_{L}$ across the surface. Also, for artery A at all times during the cardiac cycle, the non-Newtonian behaviour of blood becomes significant in a region on the inner wall at about the mid point of the artery, where the artery widens before narrowing again. (All figures can be found on the Journal's web site.) 
Another approach to assessing the importance of the non-Newtonian blood model is to consider the global non-Newtonian importance factor, $\bar{I}_{G}$, as a function of time through the cardiac cycle. Figure 8 shows the value of $\bar{I}_{G}$ as a function of time for all four arteries considered. It can be seen that for most of the cardiac cycle, using a non-Newtonian blood model is not of great significance (using the cut-off value of $\bar{I}_{G}=0.25$ (Johnston et al., 2004), the horizontal line in Figure 8). The figure shows that for all arteries the non-Newtonian model is significant for a period of about 0.2 seconds where the flow decelerates and reverses. Also, for the normal arteries, the non-Newtonian model becomes slightly significant again at the troughs in the forcing function around $t=0.43$ and $t=0.63$, while, for the ectatic artery $\mathrm{C}$, the period of time for which the non-Newtonian behaviour is significant around these points is increased and the degree of significance is increased.

\subsection{Particle Traces}

Results from the flow computations are also presented as particle plots for each artery. During the simulation, massless particles are introduced into the flow domain at the inlet. The particles are introduced at the centre of the inlet plane and in two concentric circles of differing radii surrounding the centre point. Figure 9 shows the final position of the particles after one cardiac cycle for arteries B and C for both the Newtonian and Generalised Power Law blood models (complete movie files for these simulations are available on the Journal's web site). The legend on each figure is coloured according to the time the particles have spent in the artery: blue particles are recently injected and red particles were injected at the beginning.

It is interesting to observe (from the movie files) that in most cases a majority of the particles which are injected in the beginning are removed during the reverse flow period of the cycle. This explains why there are a considerable number of green particles in Figure 9. The figure shows that there are differences firstly between the two viscosity models and secondly between 
the two arteries. Comparing the two viscosity models, it can be seen in the Generalised Power Law model that the red particles are clustered more towards the end of the artery than in the Newtonian model. This would tend to indicate that greater mixing is achieved in the Newtonian model. This is one example in which the difference between Newtonian and non-Newtonian blood models is apparent. Secondly, comparing the two arteries, in artery B the blood does not flow through the entire artery in one heart beat cycle, whereas in artery $\mathrm{C}$ some particles do exit the flow domain. Although it might be difficult to observe visually, there is a reasonable difference between the distribution of the particles when using the two blood models.

Another feature of Figure 9 is the helical pattern of the paths of the particles, especially in the proximal part of the artery. This indicates that there are significant secondary flows within the artery as the particles spiral their way through the flow domain. A careful analysis of the figure shows that there is a similar degree of spiralling between the two blood viscosity models in the proximal section of the artery. However, in the mid region of the artery the non-Newtonian model shows a lesser degree of spiralling than the Newtonian model.

\section{Discussion}

Results for transient simulation of blood flow in the human right coronary artery have been presented in the previous section. Four different arteries and two different models for blood viscosity have been considered. Results have been presented in terms of wall shear stress distribution, non-Newtonian importance factors and particle path simulations.

The idea behind this study was to assess the difference between blood flow in different coronary arteries and to compare the use of Newtonian and non-Newtonian blood viscosity models.

In a recent study on steady simulations in the same coronary arteries (Johnston et al., 2004), 
it was concluded that there was significant inter-individual variation in the wall shear stress distributions and that using a non-Newtonian model for blood viscosity was only important at low inlet velocities. In terms of the transient simulations, there is still a significant variation between the different coronary arteries in terms of wall shear stress distribution and these differences are maintained for most of the cardiac cycle. In most cases, regions of low wall shear stress predominate at the proximal end of the artery with further areas of low wall shear stress decreasing in size and frequency as the artery is traversed. This is in agreement with results presented by Kirpalani et al. (Kirpalani et al., 1999) and Myers et al. (Myers et al., 2001). The reason for this is probably related to the fact that most of the arteries taper towards the distal end. One exception is artery A which shows a region of low wall shear stress (for most of the cardiac cycle) about its mid region where the radius increases for a short distance. It is also worth commenting on the ectatic artery C. Here, there are quite intense regions of low wall shear stress near the distal end of the artery, which are probably due to the overall larger diameter of the artery along its entire length, compared with the normal arteries.

It is also worth comparing the wall shear stress distributions in Figure 6 to those obtained from steady state simulations (Johnston et al., 2004). The distributions of wall shear stress found in the steady state simulations are qualitatively the same as those obtained here for a majority of the cardiac cycle. The wall shear stress patterns differ most significantly when the flow is either slow or reversing, which is perhaps not surprising. This is in agreement with experimental observations (Asakura and Karino, 1990) as well as previous simulations which suggest that cycle averaged wall shear stress patterns are in good agreement with steady flow patters (Myers et al., 2001; van de Vosse et al., 2001; Feldman et al., 2002). Simulations presented here suggest that this is a reasonable approximation for about $70 \%$ of the cardiac cycle, ie from $t=0.30$ to $t=1.00$, ignoring the slight bumps around $t=0.43$ and $t=0.63$.

Comparing Newtonian and non-Newtonian blood viscosity models shows that, visually, 
there is little difference between wall shear stress distributions at the points of the cardiac cycle shown in Figure 5. This is further borne out in Figure 7 which shows that the non-Newtonian importance factors are only significant during periods where the blood flow is quite slow. The insets in the panels of Figure 7, corresponding to the insets in Figure 5, contain regions where the shear stress is low for the entire cardiac cycle. Even at this point of the artery, there is no strong indication that the non-Newtonian blood viscosity model is significant. However, the question is, over a complete cardiac cycle, how significant is the non-Newtonian behaviour of blood? From Figure 8 it can be seen that for all but about $30 \%$ of the period of one heart beat the difference between Newtonian and non-Newtonian blood models is not significant.

The above figure of $30 \%$ of the cardiac cycle is observed for normal arteries. In the one ectatic artery considered, the period of significance of the non-Newtonian blood model increases to about $40 \%$. However, the overall degree of significance is much decreased, being approximately one third of the value for artery D. This highlights another difference between normal and ectatic coronary arteries.

In order to examine the question of the importance of the non-Newtonian blood model from another point of view, it is worth considering Figure 10 which shows the wall shear stress distribution in artery $\mathrm{B}$ at $t=0.18$. This is the point in the cardiac cycle where the difference between the two blood models is the most significant. Note that the scale on these plots is from 0 to 0.14 , instead of the range of 0 to 4 in Figure 5. Visually, there is a strong correlation between the two distributions, with the main difference being the magnitude of the wall shear stress. In other words, regions of low wall shear stress are predicted in similar positions in both models. Note, however, the regions of "high" wall shear stress are not very high once the scale of the figure is taken into account. Hence, although there is a difference in wall shear stress distribution at this point of the cardiac cycle, it could be argued that it is insignificant given the low overall magnitudes of the wall shear stress compared to other points in the cardiac 
cycle.

Based on the above discussions, it could be concluded that using a Newtonian model for blood viscosity is an adequate approximation. This conclusion is based on consideration of the wall shear stress distribution observed during the cardiac cycle and could be valid even during slow flow periods.

On the other hand, a consideration of the particle paths, Figure 9, yields a different conclusion. Clearly, the existence of several red particles in the mid regions of the arteries for the Newtonian models for the two arteries $\mathrm{B}$ and $\mathrm{C}$ implies a difference between the internal flow patterns with the two viscosity models. For the Generalised Power Law blood model, many particles which enter the flow domain during the forward flow phase, from $t=0.0$ to $t=0.08$ seconds, are removed during the reverse flow period, from $t=0.08$ to $t=0.18$ seconds. This is not the case for the Newtonian model. A possible reason for this difference is that the Newtonian model would have a higher centreline velocity (beyond the entrance length) and so particles have the opportunity to penetrate further into the flow domain. Then, if during the reverse period they move towards the outside of the artery, where the flow is slower, they have the possibility of staying within the flow domain. The difference in the number of red particles between arteries B and C highlights another difference between the normal and ectatic arteries.

These results are also in agreement with previous results for flow in a $90^{\circ}$ bend (Gijsen et al., 1999). There it was demonstrated, both experimentally and numerically, that there was slower development of secondary flows for the non-Newtonian fluid which in turn affected the axial velocity profiles. However, the degree to which this affects the axial velocity varies with position in the artery, as shown in Figure 9.

It should be noted that Figure 9 presents a different way of representing the flow to that presented previously (Feldman et al., 2002). Here the mixing which occurs during the cardiac cycle is demonstrated by the mixture of the coloured balls within the figure. However, in 
(Feldman et al., 2002), particle traces at instances in time are given to show the complex nature of the flow.

\section{Conclusion}

This paper has presented a transient study of blood flow in four different human right coronary arteries with two different blood viscosity models.

A comparison of the four coronary arteries shows that the wall shear stress distribution forms complex patterns on the artery wall which change considerably during the cardiac cycle. However, for all arteries it appears that low wall shear stress predominates near the proximal end of the artery for a large proportion of the cardiac cycle. Higher wall shear stress exists further down the artery, particularly associated with bends and narrowing of the artery. Sudden expansions yield low wall shear stress for most of the cardiac cycle.

Comparing the two blood viscosity models shows that the non-Newtonian model is only significant for approximately $30 \%$ of the cardiac cycle (Figure 8). During these periods, the wall shear stress is very low, with the regions of lowest wall shear stress in the same positions for both models. Hence, it could be concluded that a Newtonian model for blood viscosity is an adequate approximation for transient simulations. This agrees with the conclusion of Ballyk et al. (Ballyk et al., 1994) and is despite the fact that regions of slow flow exist at various points of the cardiac cycle where non-Newtonian behaviour should be important (Pedley, 1980).

These conclusions are based on simulations for a heart rate of only 61 beats per minute (a resting rate) with only low inlet velocities (maximum $0.214 \mathrm{~ms}^{-1}$ ). If the heart rate were elevated to a moderate exercise rate of about 100 beats per minute and/or the inlet velocity increased, then the period over which the non-Newtonian blood model was significant would decrease. 
Finally, it would appear that in terms of wall shear stress distributions, and consequently in terms of global non-Newtonian importance factors, that there is little practical difference between a Newtonian and a non-Newtonian model for blood viscosity. However, analysis of the particle paths suggests that within the flow there are differences which do not greatly affect the wall shear stress distribution. Hence, for simulation purposes, to study wall shear stress a Newtonian model would be sufficient, but to study the flow in more detail, to look at mixing within the blood or stresses on individual blood cells (haemolysis) for example, the non-Newtonian behaviour of the blood should be included.

These conclusions are presented under the assumption that a fixed mesh is used to study the flow. There may be some discrepancy if the simulations were performed on a model with elastic arterial walls and the artery was allowed to move with the beating heart. 


\section{References}

Asakura, T. and Karino, T. (1990). Flow patterns and spatial distribution of atherosclerotic lesions in human coronary arteries. Circ. Res., 66:1054-1066.

Ballyk, P. D., Steinman, D. A., and Ethier, C. R. (1994). Simulation of non-Newtonian blood flow in an end-to-end anastomosis. Biorheology, 31(5):565-586.

Berger, S. A. and Jou, L.-D. (2000). Flows in stenotic vessels. Annu. Rev. Fluid Mech., 32:347382.

Berthier, B., Bouzerar, R., and Legallais, C. (2002). Blood flow patterns in an anatomically realistic coronary vessel: influence of three different reconstruction methods. Journal of Biomechanics, 35:1347-1356.

Caro, C. G. (2001). Vascular fluid dynamics and vascular biology and disease. Mathematical Methods in the Applied Sciences, 24:1311-1324.

Caro, C. G., Fitz-Gerald, J. M., and Schroter, R. C. (1971). Atheroma and arterial wall shear: observation, correlation and proposal of a shear dependent mass transfer mechanism for atherogenesis. Proc. R. Soc. Lond., B177:109-159.

Cho, Y. I. and Kensey, K. R. (1991). Effects of the non-Newtonian viscosity of blood on flows in a diseased arterial vessel. Part 1: Steady flows. Biorheology, 28:241-262.

Corney, S., Johnston, P. R., and Kilpatrick, D. (2001). Cyclic flow patterns in human coronary arteries. In Computers in Cardiology, pages 21-24. IEEE, IEEE Press.

Corney, S., Johnston, P. R., and Kilpatrick, D. (2004). Construction of realistic branched, threedimensional arteries suitable for computational modelling of flow. Medical and Biological Engineering and Computing, 42:660-668. 
Feldman, C. L., Ilegbusi, O. J., Hu, Z., Nesto, R., Waxman, S., and Stone, P. H. (2002). Determination of in vivo velocity and endothelial shear stress patterns with phasic flow in human coronary arteries: A methodology to predict progression of coronary athersclerosis. American Heart Journal, 143:931-939.

Fry, D. (1968). Acute vascular endothelial changes associated with increased blood velocity gradients. Circ. Res., 22:165-197.

Fung, Y. C. (1993). Biomechanics: Mechanical Properties of Living Tissues. Springer-Verlag, 2nd edition.

Giddens, D. P., Zarins, C. K., and Glagov, S. (1990). Response of arteries to near wall fluid dynamic behavior. Applied Mechanics Review, 43 (2):S98-S102.

Gijsen, F. J. H., Allanic, E., van de Vosse, F. N., and Janssen, J. D. (1999). The influence of the non-Newtonian properties of blood on the flow in large arteries: unsteady flow in a $90^{\circ}$ curved tube. Journal of Biomechanics, 32:705-713.

Johnston, B. M., Johnston, P. R., Corney, S., and Kilpatrick, D. (2004). Non-Newtonian blood flow in human right coronary arteries: Steady state simulations. Journal of Biomechanics, $37(5): 709-720$.

Kirpalani, A., Park, H., Butany, J., Johnston, K. W., and Ojha, M. (1999). Velocity and wall shear stress patterns in the human right coronary artery. Journal of Biomechanical Engineering, 121:370-375.

Krams, R., Wentzel, J. J., Oomen, J. A. F., Vinke, R., H., J. C., Schuurbiers, de Feyter, P. J., Serruys, P. W., and Slager, C. J. (1997). Evaluation of endothelial shear stress and 3D geometry as factors determining the development of atherosclerosis and remodelling in 
human coronary arteries in vivo - combining 3D reconstruction from angiography and ivus (angus) with computational fluid dynamics. Arteriosclerosis, Thrombosis and Vascular Biology, 17:2061-2065.

Ku, D., Giddens, D., Zarins, C., and Glagov, S. (1985). Pulsatile flow and atherosclerosis in the human carotid bifurcation: Positive correlation between plaque and low and oscillating shear stress. Arteriosclerosis, 5:293-302.

Liepsch, D. (2002). An introduction to biofluid mechanics — basic models and applications. Journal of Biomechanics, 35:415-435.

Matsuo, S., Tsuruta, M., Hayano, M., Immamura, Y., Eguchi, Y., Tokushima, T., and Tsuji, S. (1988). Phasic coronary artery flow velocity determined by Doppler flowmeter catheter in aortic stenosis and aortic regurgitation. The Americal Journal of Cardiology, 62(1):917922.

Myers, J. G., Moore, J. A., Ojha, M., Johnston, K. W., and Ethier, C. R. (2001). Factors influencing blood flow patterns in the human right coronary artery. Annals of Biomedical Engineering, 29:109-120.

Ojha, M., Leask, R. L., Butany, J., and Johnston, K. W. (2001). Distribution of intimal and medial thickening in the human right coronary artery: a study of 17 RCAs. Atherosclerosis, 158:147-153.

Pedley, T. J. (1980). The fluid mechanics of large blood vessels. Cambridge University Press.

Perktold, K., Peter, R., and Resch, M. (1989). Pulsatile non-Newtonian blood flow simulation through a bifurcation with an aneurism. Biorheology, 26:1011-1030.

Perktold, K., Peter, R. O., Resch, M., and Langs, G. (1991a). Pulsatile non-Newtonian blood 
flow in three-dimensional carotid bifurcation models: a numerical study of flow phenomena under different bifurcation angles. J. Biomed. Eng., 13:507-515.

Perktold, K. and Resch, M. (1990). Numerical flow studies in human carotid artery bifurcations: basic discussion of the geometry factor in atherogenesis. J. Biomed. Eng., 12:111-123.

Perktold, K., Resch, M., and Peter, R. O. (1991b). Three-dimensional numerical analysis of the pulsatile flow and wall shear stress in the carotid artery bifurcation. J. Biomechanics, 24(6):409-420.

Rodkiewicz, C. M., Sinha, P., and Kennedy, J. S. (1990). On the application of a constitutive equation for whole human blood. Journal of Biomechanical Engineering, 112:198-206.

Tu, C. and Deville, M. (1996). Pulsatile flow of non-Newtonian fluids through arterial stenoses. J. Biomechanics, 29(7):899-908.

van de Vosse, F. N., Gijsen, F. J. H., and Wolters, B. J. B. M. (2001). Numerical analysis of coronary artery flow. In 2001 Bioengineering Conference, volume 50, pages 17-18. ASME.

van Langenhove, G., Wentzel, J. J., Krams, R., Slager, C. J., Hamburger, J. N., and Serruys, P. W. (2000). Helical velocity patterns in a human coronary artery. Circulation, 102:e22$\mathrm{e} 24$.

Walburn, F. J. and Schneck, D. J. (1976). A constitutive equation for whole human blood. Biorheology, 13:201-210.

Wentzel, J. J., Gijsen, F. J. H., Stergiopulos, N., Seruys, P. W., Slager, C. J., and Krams, R. (2003). Shear stress, vascular remodeling and neointimal formation. Journal of Biomechanics, 36:681-688. 
Zeng, D., Ding, Z., Friedman, M. H., and Ethier, C. R. (2003). Effects of cardiac motion on right coronary artery hemodynamics. Annals of Biomedical Engineering, 31:420-429.

Zhu, H., Warner, J. J., Gehrig, T. R., and Friedman, M. H. (2003). Comparison of coronary artery dynamics pre- and post-stenting. Journal of Biomechanics, 36:689-697. 


\section{Figure Captions}

Figure 1: Angiogram pictures, taken at the same point in the cardiac cycle from two different views, used to reconstruct the computational mesh for artery B. The upper and lower crosses in the frames indicate the beginning (upper) and end (lower) of the artery used for the simulation.

Figure 2: Arterial radius as a function of arc-length for each of the four arteries.

Figure 3: Computational mesh for artery B used for the simulations.

Figure 4: Physiological forcing function used to drive the inlet velocity boundary condition as a function of time (Matsuo et al., 1988, Figure 4A).

Figure 5: Wall shear stress distributions (in Pascal) for the Generalised Power Law blood model in artery $\mathrm{B}$ at various points of the cardiac cycle. The inset figures in the upper right corner of each panel show the wall shear stress distribution, in the section of the artery indicated by the box, from the reverse direction.

Figure 6: Wall shear stress distributions (in Pascal) for the Generalised Power Law blood model in each of the arteries at $t=0.85$ seconds, the point of maximum flow during the cardiac cycle. The format is the same as in Figure 5.

Figure 7: Distribution of the local non-Newtonian importance factor in artery B at various points of the cardiac cycle. The format is the same as in Figure 5.

Figure 8: Global non-Newtonian importance factors for each of the four arteries during the cardiac cycle.

Figure 9: Final particle positions in arteries $\mathrm{B}$ and $\mathrm{C}$ for the Newtonian and Generalised Power Law blood models.

Figure 10: Wall shear stress distributions (in Pascal) in artery B at the point in the cardiac cycle when the non-Newtonian blood model has greatest significance. The format is the same as in Figure 5. 


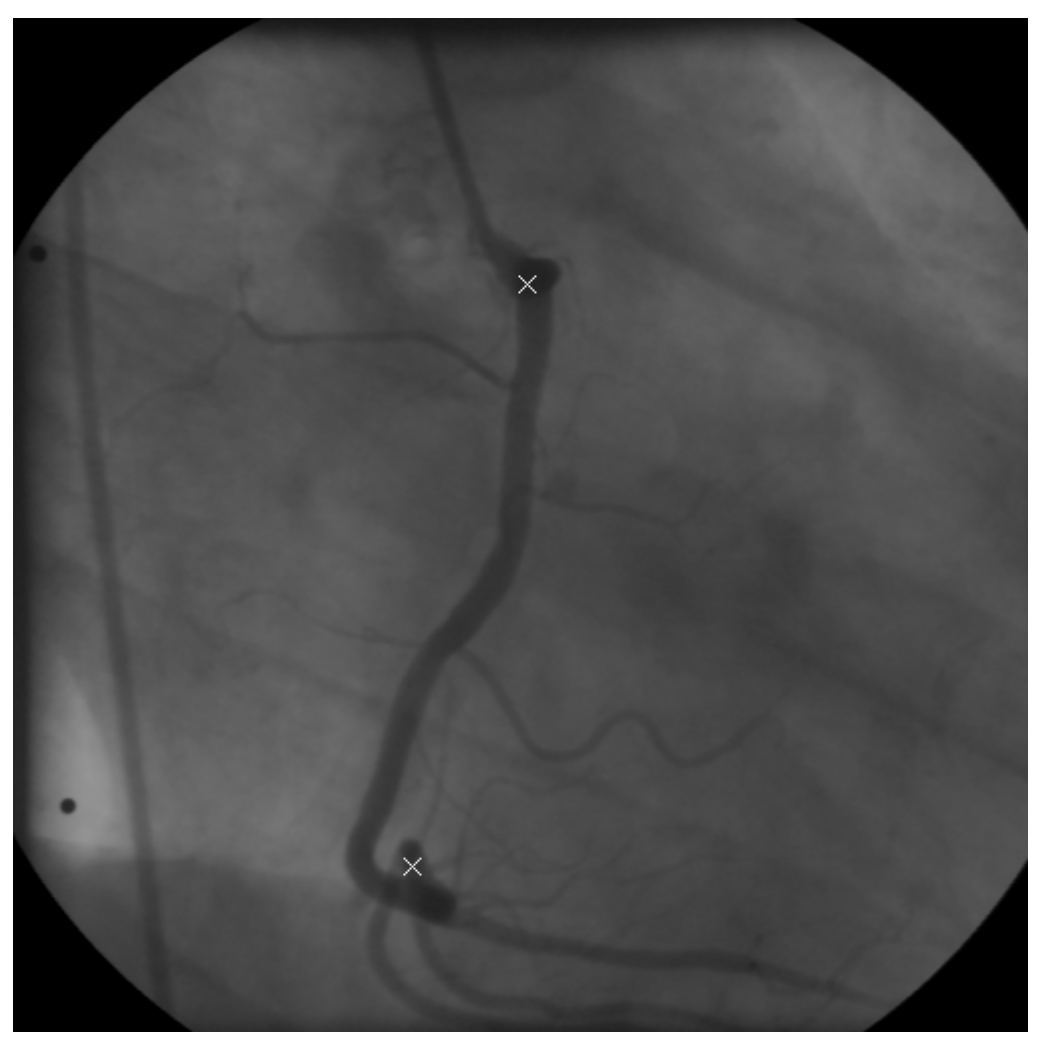

(a) Frame 15

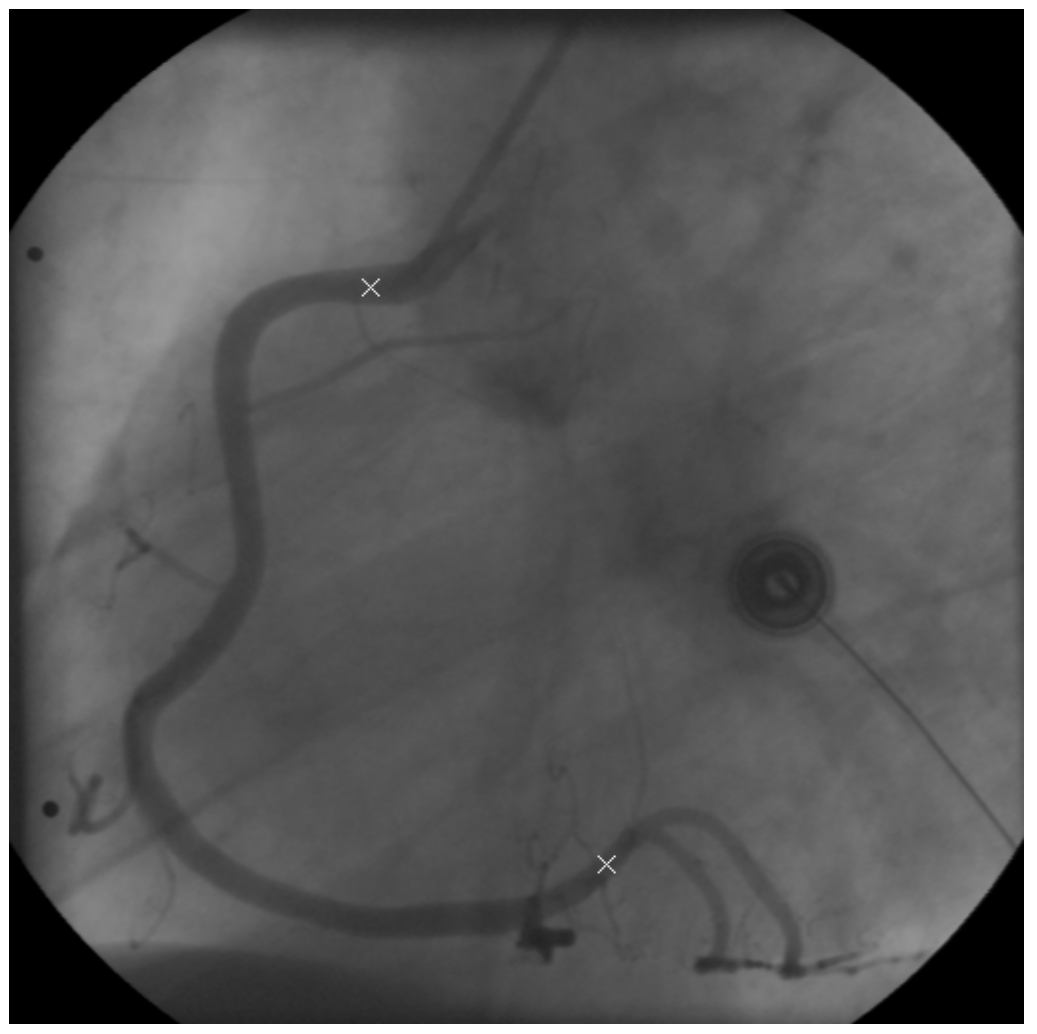

(b) Frame 16

Figure 1: 


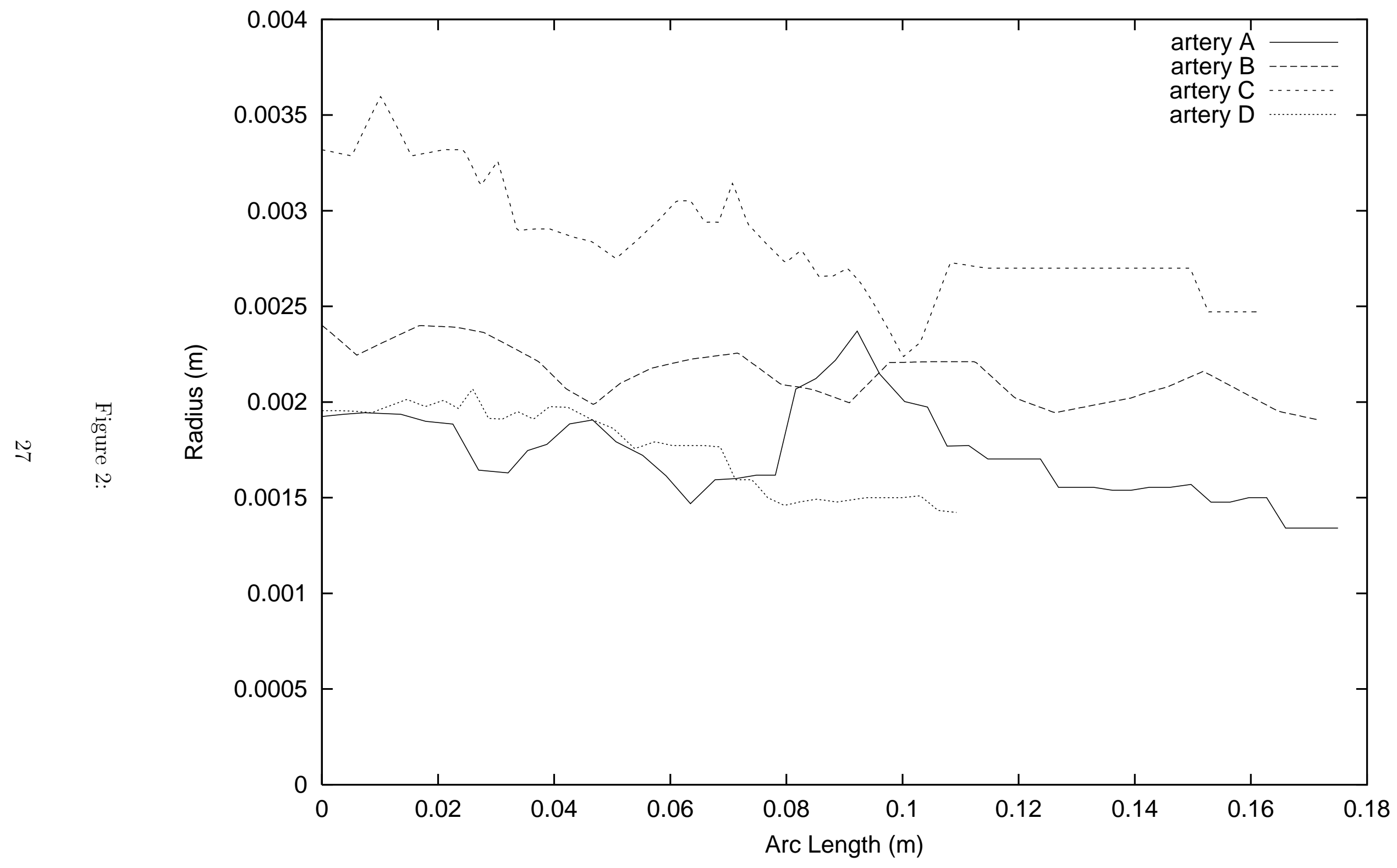




$$
\mathcal{L}
$$




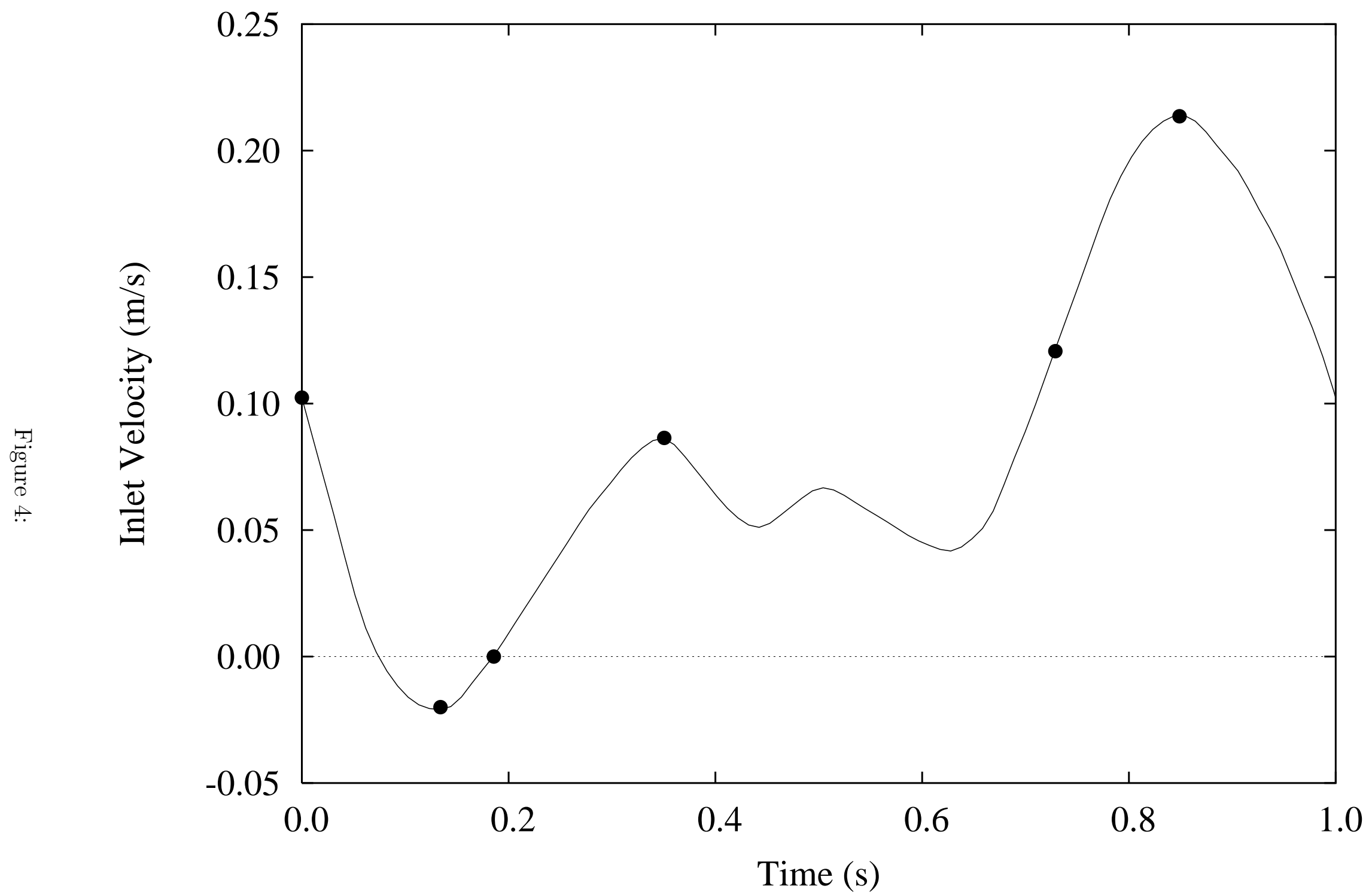



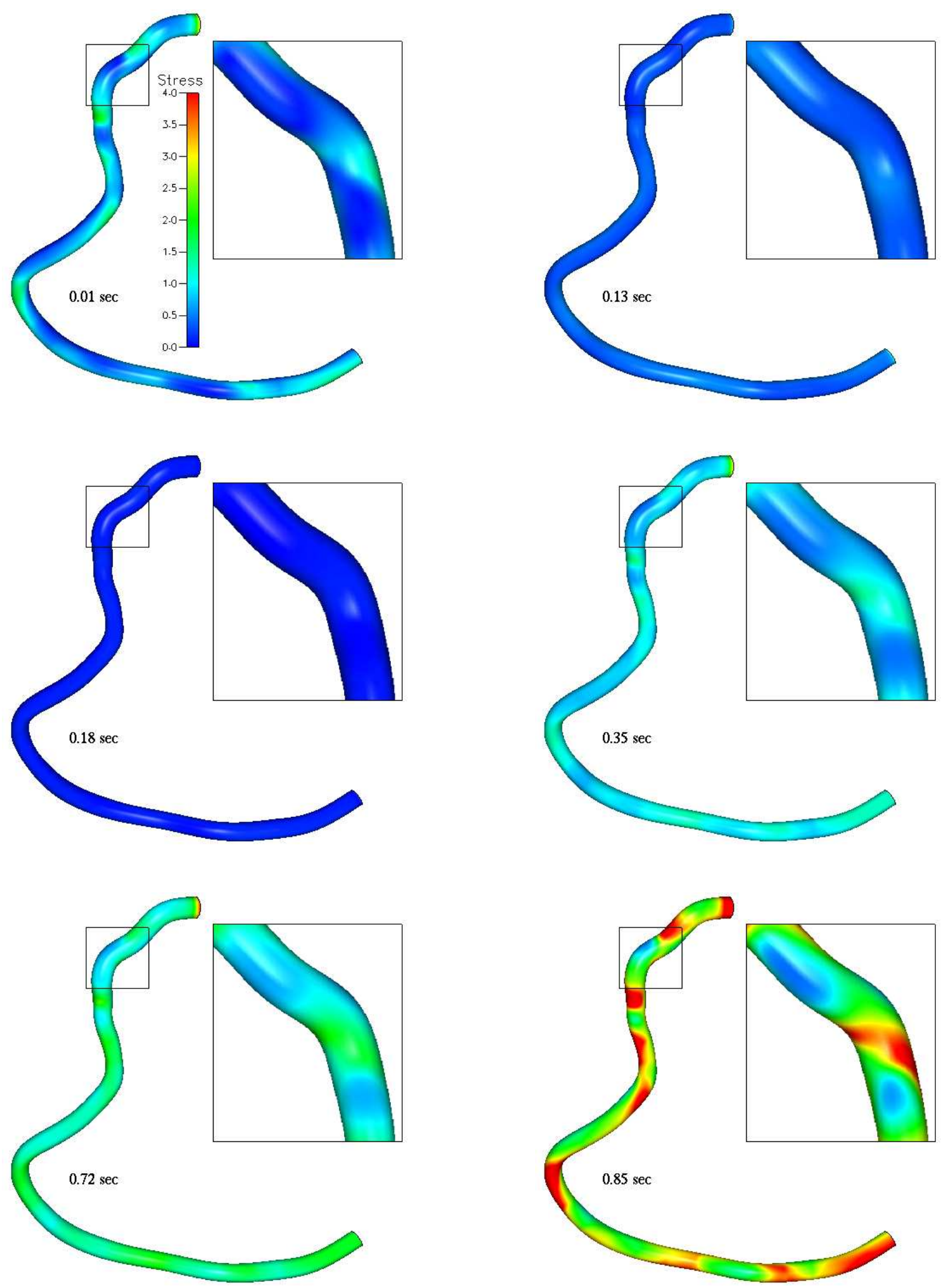

Figure 5: 


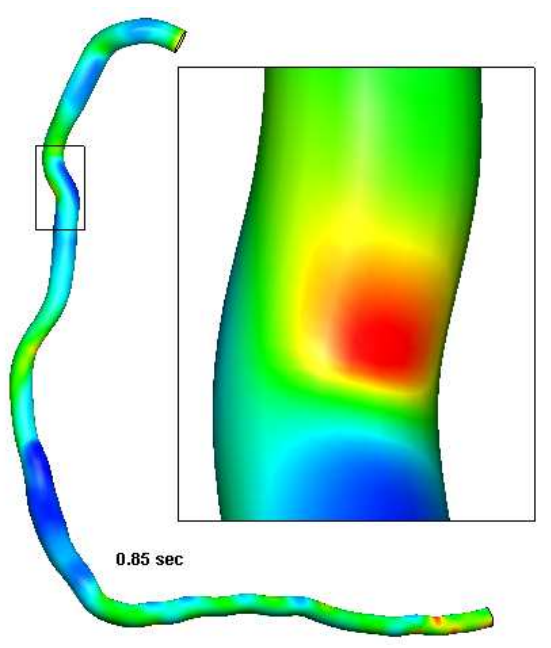

Artery A

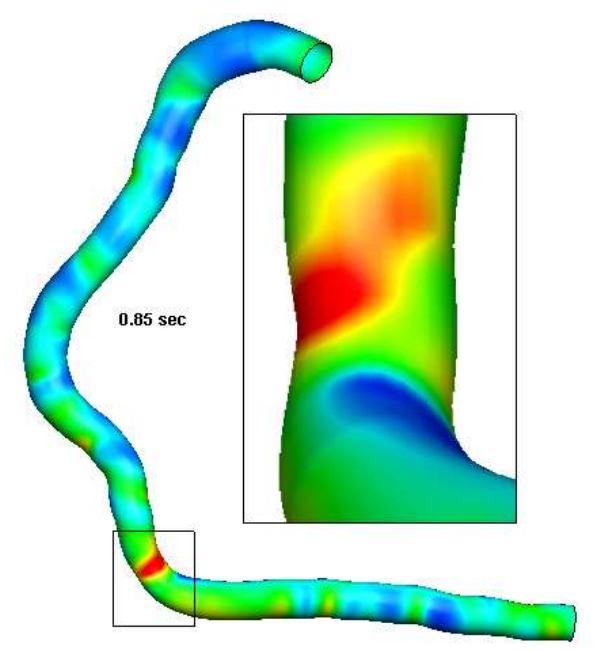

Artery C

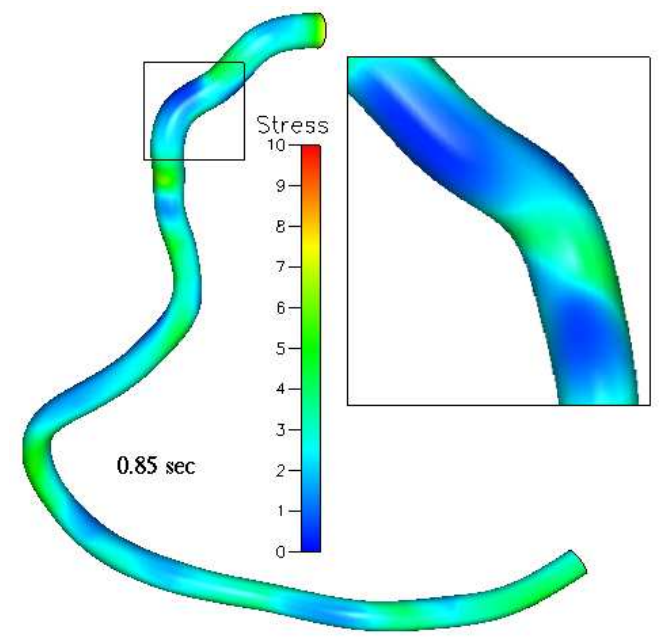

Artery B

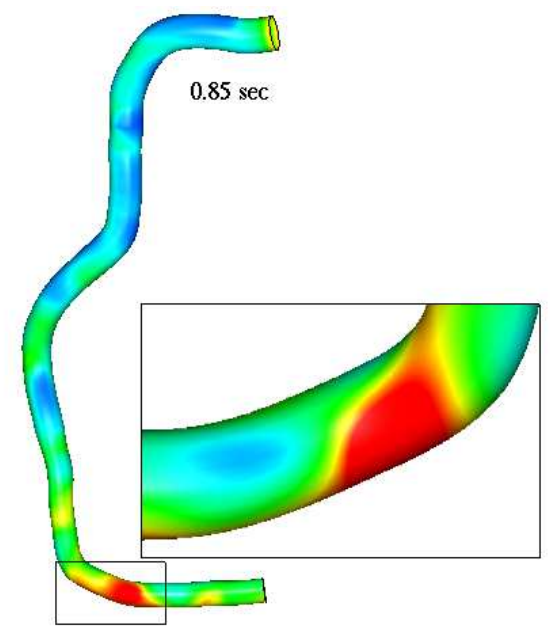

Artery D

Figure 6: 

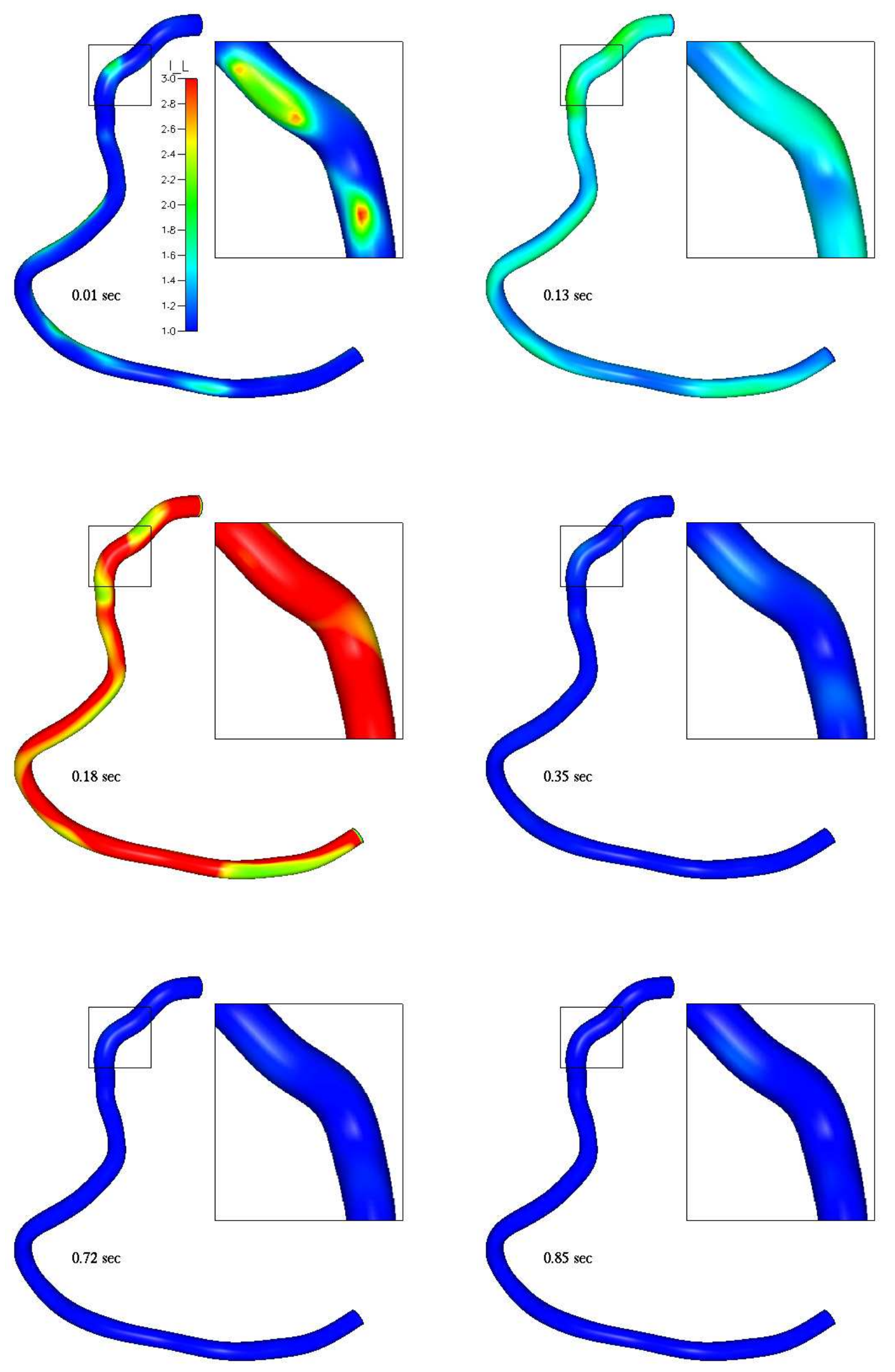

Figure 7: 


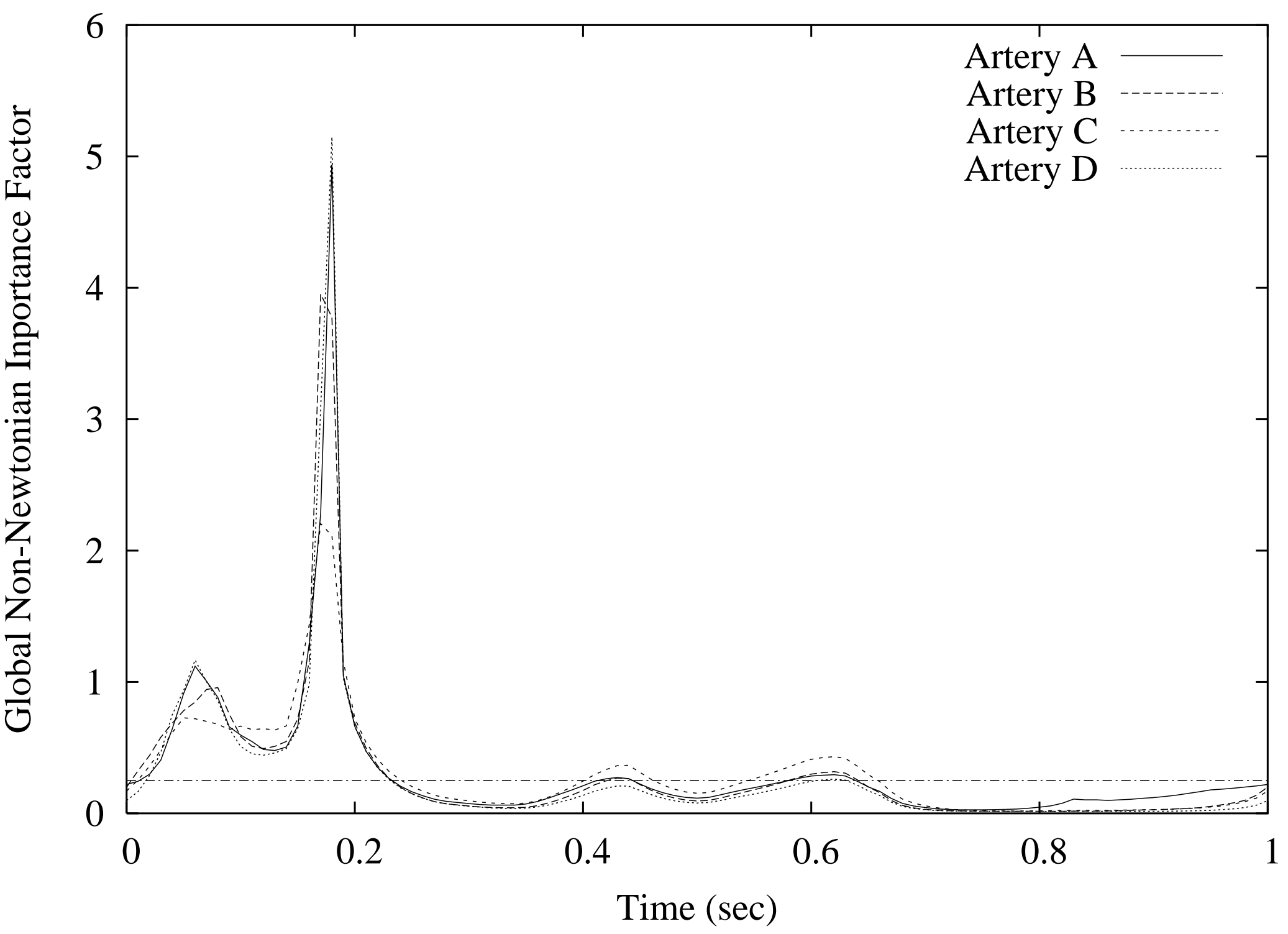




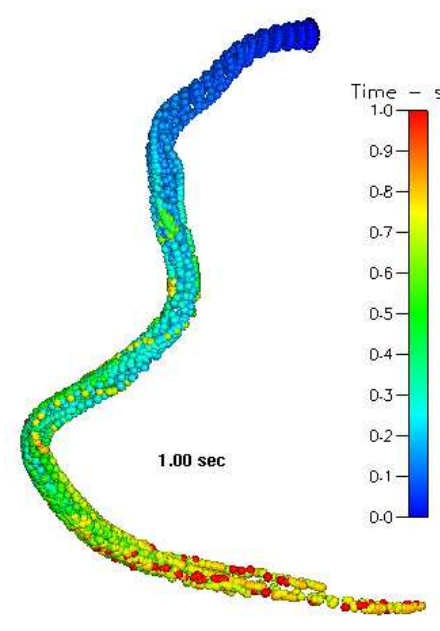

(a) Artery B, Newtonian

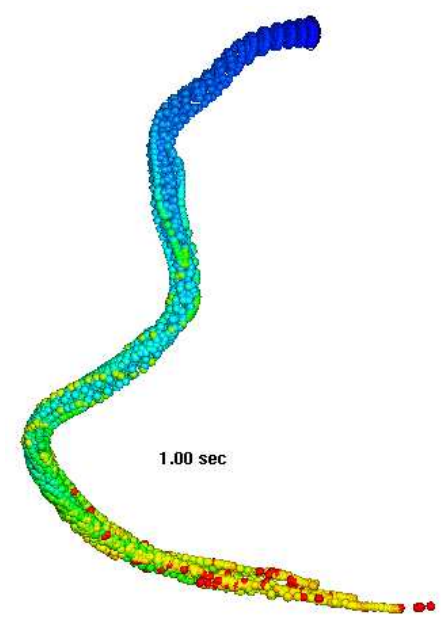

(c) Artery B, Generalised Power Law

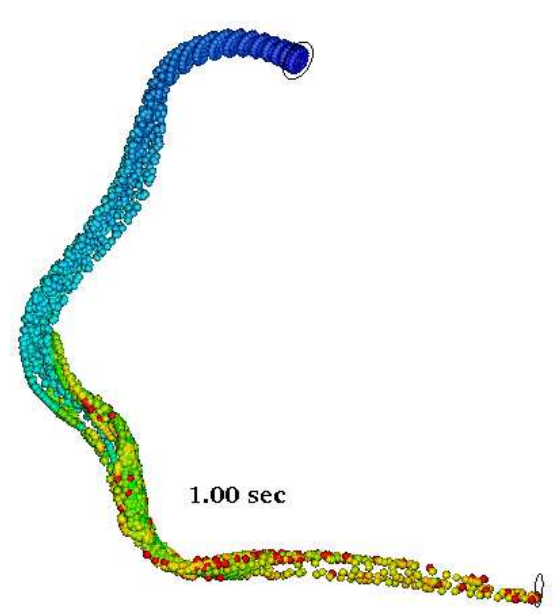

(b) Artery C, Newtonian

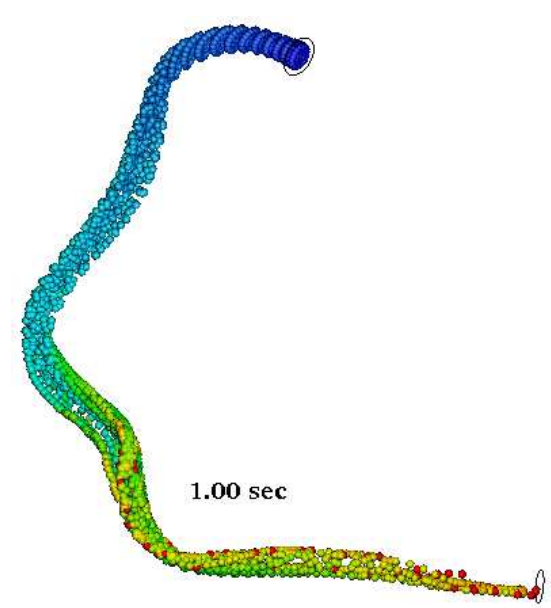

(d) Artery C, Generalised Power Law

Figure 9: 


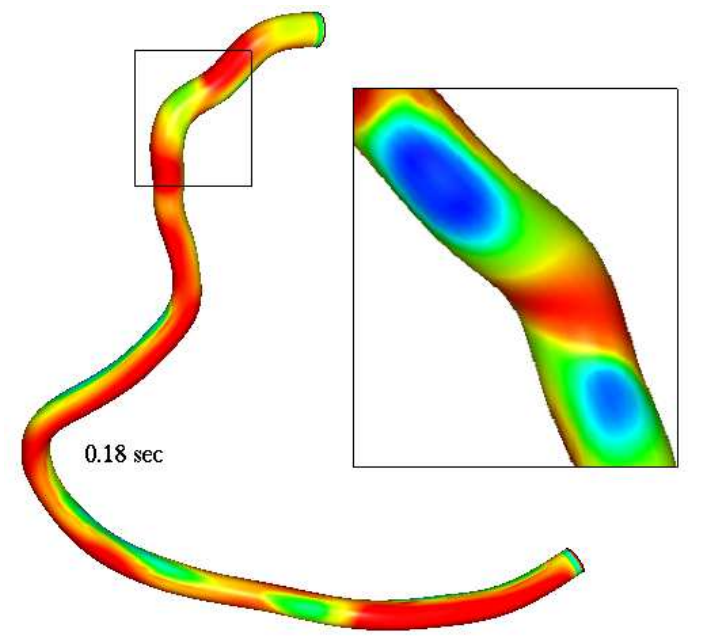

Newtonian

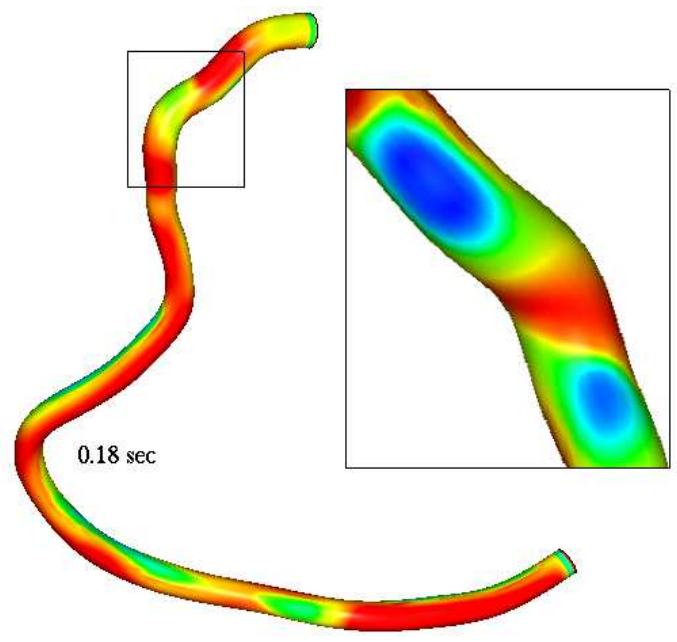

Generalised Power Law

Figure 10: 